\title{
p53, mdm2, EGFR, and msh2 expression in paired initial and recurrent glioblastoma multiforme
}

\author{
A M Stark, P Witzel, R J Strege, H-H Hugo, H M Mehdorn
}

J Neurol Neurosurg Psychiatry 2003;74:779-783

See end of article for authors' affiliations

......................

Correspondence to:

Dr Andreas M Stark,

Department of

Neurosurgery, University of

Kiel Medical Centre,

Weimarer Str 8, D-24106

Kiel, Germany;

andstark@hotmail.com

Received 21 September

2002

In revised form

15 December 2002

Accepted

27 December 2002

Background: The clinical course of glioblastoma multiforme is characterised by invasive growth and regular recurrence. Many genetic alteration have been identified in the genesis of the disease. However, information about immunohistochemical expression in recurrent lesions is sparse.

Objectives: To determine (1) whether the $\mathrm{p} 53 / \mathrm{mdm} 2 / E G F R / m s h 2$ expression pattern differs in initial $v$ recurrent glioblastoma multiforme; (2) whether a possible change in expression correlates with prognostic variables (progression-free survival time, total survival time); and (3) whether chemotherapy in addition to surgery and radiotherapy influences the $\mathrm{p} 53 / \mathrm{mdm} 2 / \mathrm{EGFR} / \mathrm{msh} 2$ expression profile.

Methods: 27 patients were studied. They met the following criteria: histologically confirmed diagnosis of glioblastoma multiforme (WHO IV); total tumour resection at initial craniotomy; at least one re-craniotomy for glioblastoma multiforme recurrence; age 21 years or older. All underwent radiotherapy of at least $54 \mathrm{~Gy}$, and 17 received additional chemotherapy. Immunohistochemical staining of initial tumours and recurrences was done with the following monoclonal antibodies: anti-p53 (DO-1), anti-mdm2 (IF-2), anti-EGFR (H1 1), and anti-msh2 (AB-1).

Results: In comparison with the initial tumour, recurrent lesions were characterised by reduced expression of $p 53(p<0.0001)$ and msh2 ( $=0.0012)$, while the numbers of mdm2 ( $p=0.02)$, EGFR $(p<0.0001)$, and msh2 positive specimens $(p<0.0001)$ were reduced. Chemotherapy was associated with reduced msh2 expression ( $<0.0001)$. Immunohistochemical variables were not associated with patient survival.

Conclusions: There are significant differences in the $\mathrm{p} 53 / \mathrm{mdm} 2 /$ EGFR/msh2 expression patterns in initial $v$ recurrent glioblastoma multiforme. There may be interactions between chemotherapy and changes in the msh2 expression.

G ioblastoma multiforme is characterised by hypercellularity, nuclear polymorphism, brisk mitotic activity, prominent microvascular proliferation, and necrosis. Many genetic alterations have been detected in this tumour, the most common involving the tumour suppressor gene TP53, the cellular oncogene MDM2, and the epidermal growth factor receptor (EGFR). ${ }^{1-5}$ Mutations in the TP53 gene are typical events in primary glioblastoma multiforme, which is characterised by a short clinical history (usually less than three months) and the absence of a pre-existing, less malignant astrocytoma. In contrast, $\mathrm{mdm} 2$ and EGFR protein overexpression are characteristic features of secondary glioblastoma multiforme, which progresses from less malignant astrocytoma. $^{5-8}$

In response to cellular stress, p53 protein leads to cell cycle arrest or even apoptosis in order to prevent the replication of damaged DNA. Thus p53 prevents malignant growth. The key regulator of $\mathrm{p} 53$ is the oncoprotein $\mathrm{mdm} 2$, which can lead to p53 inactivation and shuttling into the cytoplasm, where p53 cannot activate its downstream factors. ${ }^{10}$ Following its activation, the EGF receptor initiates a transforming autocrine loop, thus contributing to cell division. ${ }^{1}$ The msh2 protein is part of the DNA mismatch repair system (MMR). Defects in the MMR have not only been detected in many human tumours but have also been shown to correlate with the response of glioblastoma multiforme to methylating agents. ${ }^{11-13}$ However, none of these factors has yet been considered to be of independent prognostic value. ${ }^{11415}$

Information about the $\mathrm{p} 53, \mathrm{mdm} 2$, EGFR, and msh2 expression in recurrent glioblastoma multiforme is sparse. This study was designed to answer the following questions: does the p53/ $\mathrm{mdm} 2 / \mathrm{EGFR} / \mathrm{msh} 2$ expression pattern differ in initial $v$ recurrent glioblastoma multiforme; does a possible change in expres- sion correlate with prognostic variables (progression-free survival time, total survival time); and does the application of chemotherapy in addition to surgery and radiotherapy influence the $\mathrm{p} 53 / \mathrm{mdm} 2 / \mathrm{EGFR} / \mathrm{msh} 2$ expression profile?

\section{METHODS}

\section{Patient data}

Between 1994 and 2000, 144 patients underwent craniotomy for newly diagnosed glioblastoma multiforme in our department. Forty three patients $(29.9 \%)$ underwent at least one re-craniotomy for a recurrence of the tumour. The criteria for inclusion in the study were as follows:

- histological diagnosis of glioblastoma (WHO IV) without a significant oligodendrial component;

- total resection of the initial tumour, verified by the absence of a contrast enhancing region in the early postoperative computed tomography (CT) (done within 12 to 24 hours after surgery);

- patient's age 21 years or older.

Twenty seven patients met these criteria ( 17 male, 10 female; median age 56 years, range 29 to 73 ). Seven patients underwent re-craniotomy for second tumour recurrence. All lesions were classified as primary glioblastoma multiforme because the onset of the disease was less than three months before diagnosis and there was no history of previously detected less malignant astrocytoma. The primary nature of the tumours was not confirmed by genetic testing.

All patients received postoperative irradiation of at least 54 Gy. Seventeen underwent chemotherapy (cisplatin/tamoxifen (14), gliadel (2), temozolomide for glioblastoma multiforme recurrence (1)). All tumours were located in the cerebral 
Table 1 Patient data (progression-free survival time and total survival time in days)

\begin{tabular}{|c|c|c|c|c|c|c|c|c|c|c|c|c|c|}
\hline $\begin{array}{l}\text { Patient } \\
\text { No }\end{array}$ & $\begin{array}{l}\text { Age } \\
\text { (years) }\end{array}$ & Sex & Chemo & p53/i & $\mathrm{p} 53 / \mathrm{R}$ & $\mathrm{mdm} 2 / \mathrm{i}$ & $\mathrm{mdm} 2 / \mathrm{R}$ & EGFR/i & EGFR/R & $\mathrm{msh} 2 / \mathrm{i}$ & $\mathrm{msh} 2 / \mathrm{R}$ & PFST & TST \\
\hline 1 & 65 & Male & Yes & $61 \%$ & $19 \%$ & $9 \%$ & $15 \%$ & + & + & $56 \%$ & $50 \%$ & 163 & 273 \\
\hline 2 & 73 & Male & Yes & $36 \%$ & $4 \%$ & $5 \%$ & $17 \%$ & + & - & $53 \%$ & $32 \%$ & 198 & 279 * \\
\hline 3 & 66 & Male & No & $11 \%$ & $31 \%$ & $60 \%$ & $0 \%$ & + & - & $88 \%$ & $61 \%$ & 194 & 556 \\
\hline 4 & 60 & Female & No & $73 \%$ & $8 \%$ & $17 \%$ & $16 \%$ & + & - & $32 \%$ & $55 \%$ & 157 & $157^{*}$ \\
\hline 5 & 64 & Female & No & $56 \%$ & $12 \%$ & $27 \%$ & $17 \%$ & + & - & $82 \%$ & $63 \%$ & 146 & 189 \\
\hline 6 & 66 & Female & Yes & $26 \%$ & $10 \%$ & $11 \%$ & $39 \%$ & + & + & $68 \%$ & $45 \%$ & 98 & 399 \\
\hline 7 & 62 & Female & Yes & $14 \%$ & $3 \%$ & $15 \%$ & $51 \%$ & + & + & $75 \%$ & $45 \%$ & $\dagger$ & $\dagger$ \\
\hline 8 & 63 & Male & Yes & $18 \%$ & $3 \%$ & $10 \%$ & $1 \%$ & - & - & $66 \%$ & $59 \%$ & 131 & 819 \\
\hline 9 & 63 & Male & No & $14 \%$ & $35 \%$ & $18 \%$ & $39 \%$ & + & - & $54 \%$ & $45 \%$ & 63 & 298 \\
\hline 10 & 56 & Female & Yes & $23 \%$ & $9 \%$ & $19 \%$ & $2 \%$ & + & + & $24 \%$ & $57 \%$ & 270 & 1109 \\
\hline 11 & 50 & Male & No & $67 \%$ & $14 \%$ & $17 \%$ & $13 \%$ & + & - & $58 \%$ & $60 \%$ & 604 & 620 \\
\hline 12 & 51 & Male & no & $36 \%$ & $12 \%$ & $15 \%$ & $6 \%$ & + & - & $67 \%$ & $40 \%$ & 635 & 1113 \\
\hline 13 & 47 & Male & Yes & $33 \%$ & $11 \%$ & $2 \%$ & $1 \%$ & + & - & $71 \%$ & $51 \%$ & 99 & 255 \\
\hline 14 & 58 & Male & Yes & $44 \%$ & $31 \%$ & $5 \%$ & $1 \%$ & + & + & $69 \%$ & $37 \%$ & 82 & 293 \\
\hline 15 & 59 & Male & Yes & $39 \%$ & $0 \%$ & $8 \%$ & $1 \%$ & + & $\dagger$ & $82 \%$ & $51 \%$ & 238 & 321 \\
\hline 16 & 53 & Male & Yes & $21 \%$ & $30 \%$ & $15 \%$ & $19 \%$ & - & - & $71 \%$ & $52 \%$ & 89 & 305 \\
\hline 17 & 50 & Male & Yes & $65 \%$ & $12 \%$ & $3 \%$ & $12 \%$ & + & + & $72 \%$ & $47 \%$ & 105 & 439 \\
\hline 18 & 55 & Male & Yes & $21 \%$ & $0 \%$ & $22 \%$ & $31 \%$ & + & - & $65 \%$ & $59 \%$ & 151 & 424 \\
\hline 19 & 29 & Female & Yes & $0 \%$ & $39 \%$ & $17 \%$ & $0 \%$ & + & - & $63 \%$ & $46 \%$ & 248 & 433 \\
\hline 20 & 29 & Female & Yes & $48 \%$ & $0 \%$ & $5 \%$ & $10 \%$ & + & + & $71 \%$ & $45 \%$ & 426 & 738 \\
\hline 21 & 32 & Male & No & $34 \%$ & $13 \%$ & $1 \%$ & $7 \%$ & + & - & $75 \%$ & $74 \%$ & 86 & 1495 \\
\hline 22 & 39 & Female & Yes & $32 \%$ & $56 \%$ & $10 \%$ & $3 \%$ & + & + & $43 \%$ & $57 \%$ & 248 & 561 \\
\hline 23 & 63 & Female & No & $1 \%$ & $27 \%$ & $12 \%$ & $0 \%$ & - & - & $36 \%$ & $58 \%$ & 139 & 309 \\
\hline 24 & 58 & Female & Yes & $0 \%$ & $33 \%$ & $9 \%$ & $5 \%$ & + & H & $23 \%$ & $46 \%$ & 190 & 400 * \\
\hline 25 & 50 & Male & Yes & $53 \%$ & $27 \%$ & $18 \%$ & $35 \%$ & + & - & $22 \%$ & $64 \%$ & $\dagger$ & 353 \\
\hline 26 & 46 & Male & No & $11 \%$ & $17 \%$ & $1 \%$ & $7 \%$ & + & + & $62 \%$ & $42 \%$ & 200 & 541 \\
\hline 27 & 49 & Male & No & $8 \%$ & $0 \%$ & $13 \%$ & $4 \%$ & + & + & $66 \%$ & $42 \%$ & 152 & 383 \\
\hline
\end{tabular}

*Last contact; †result not available or data not known.

/i, immunohistochemical expression in initial tumours; /R, immunohistochemical expression in recurrent lesions; Chemo, chemotherapy; PFST, progression-free survival time; TST, total survival time.

hemispheres. In 11 cases more than one cerebral lobe was affected, while the tumour was restricted to a single lobe in 16 cases (frontal in seven, parietal in four, temporal in five).

Twenty six patients could be followed postoperatively. In these, clinical examination and MRI studies were done every three months or when tumour recurrence was clinically suspected. Progression-free survival time was defined as the time interval between tumour resection and recraniotomy for first tumour recurrence, and could be determined in 25 patients. Total survival time was defined as the time interval between initial craniotomy and the day of the patient's death.

\section{Immunohistochemistry}

Serial $4 \mu \mathrm{m}$ slices were obtained from formalin fixed and paraffin embedded tissue specimens. Sections were dewaxed in xylene and rehydrated in alcohol followed by wet autoclave pretreatment $\left(10\right.$ minutes at $\left.120^{\circ} \mathrm{C}\right)$ in citrate buffer for antigen retrieval. p53, mdm2, and msh2 protein expression was detected using the following monoclonal antibodies $(\mathrm{mAb})$ : anti-p53 (clone DO1, Oncogene, Cambridge, Massachusetts, USA; dilution 1:800; incubation overnight at $4^{\circ} \mathrm{C}$ ); anti-mdm2 (clone IF-2, Dianova, Hamburg, Germany; dilution 1:100; incubation overnight at $4^{\circ} \mathrm{C}$ ); and anti-msh2 (clone $\mathrm{AB}-\mathrm{l}$, Oncogene; dilution 1:25; incubation one hour at $\left.37^{\circ} \mathrm{C}\right)$. Staining for EGFR was done using the $\mathrm{H} 1 \mathrm{lmAb}$ (Dako, Carpinteria, California, USA) in a dilution of 1:200 with an overnight incubation at $4^{\circ} \mathrm{C}$. The Dako envision kit ${ }^{\circledR}$ was used for primary antibody detection. Staining was done using AECchromogen, and hemalaun was used for counterstaining. p53, mdm2, EGFR, and msh2-expressing glioma specimens from earlier studies or colorectal and hepatocellular carcinomas were used as positive controls. The primary antibody was omitted for negative controls. One glioblastoma multiforme recurrence sample stained with EGFR was found to be insufficient for estimation and was excluded from the analysis.

\section{Staining classification and data analysis}

Different classification systems for p53, mdm2, and EGFR staining in glioblastoma multiforme have been described in published reports, whereas information about msh2 is sparse. We chose a system described by Newcomb et al and Velasco et al. ${ }^{16}$ Scoring results for $\mathrm{p} 53, \mathrm{mdm} 2$, and $\mathrm{msh} 2$ nuclear staining were classified as follows: 0, no staining; 1, detectable staining in less than $5 \%$ of the cells; 2 , nuclear staining of between $5 \%$ and $60 \%$ of the cells; $3,>60 \%$ of the cells stained. For statistical analysis, a p53 and mdm 2 score of 0 and 1 was regarded as negative, while a score of 2 or 3 was regarded as positive. An msh2 score of 0-2 was regarded as reduced expression, while a score of 3 was regarded as normal expression. ${ }^{11}{ }^{16}$ EGFR staining was classified as follows: -, negative; + , up to $50 \%$ of cells stained; ++ , more than $50 \%$ of cells stained. For statistical analysis, + and ++ were regarded as positive. ${ }^{18}$

In order to detect possible differences in initial $v$ recurrent tumours in the whole series, as well as with regard to the administration of chemotherapy (given $v$ not given), the following tests were used: $\chi^{2}$ testing to compare the numbers of positive specimens; the Wilcoxon test to compare immunohistochemical scoring in each group; and the log-rank test (Cox-Mantel) to detect possible associations between survival (progression-free survival time and total survival time) and the following variables: age $(<55$ years $v \geqslant 55$ years); sex; chemotherapy (given $v$ not given); Karnowski performance scale score $<80 v \geqslant 80$; Karnowski performance scale score $<90 v \geqslant 90$; immunohistochemical expression positive $v$ negative results for $\mathrm{p} 53, \mathrm{mdm} 2, \mathrm{msh} 2$, and EGFR in initial tumours; positive $v$ negative results of $\mathrm{p} 53, \mathrm{mdm} 2, \mathrm{msh} 2$, and EGFR in first recurrences; and loss of $\mathrm{p} 53, \mathrm{mdm} 2, \mathrm{msh} 2$, and EGFR expression $v$ no loss. The latter experiment was chosen because of the significant difference in the rate of immunohistochemical results or the mean immunohistochemical score in p53, mdm2, EGFR, and msh2.

\section{RESULTS}

\section{Patient characteristics}

Twenty three of the 26 patients died during the follow up, with a mean survival time of 532 days after diagnosis (76 weeks; 
Table 2 Correlation of immunohistochemical expression in 27 paired initial tumours and first recurrences

\begin{tabular}{lllll}
\hline Protein & & Initial tumours & First recurrence & $\mathrm{p}$ Value* \\
\hline $\mathrm{p} 53$ & Positive & 24 & 20 & $\mathrm{NS}$ \\
& Negative & 3 & 7 & \\
$\mathrm{mdm} 2$ & Mean score & $35 \%$ & $22 \%$ & $\mathrm{p}=0.02$ \\
& Positive & 23 & 17 & \\
& Negative & 4 & 10 & \\
EGFR & Mean score & $15 \%$ & $20 \%$ & $\mathrm{p}<0.0001$ \\
& Positive & 24 & $11 \dagger$ & \\
msh2 & Negative & 3 & 15 & $\mathrm{p}<0.0001$ \\
& Positive & 17 & 5 & \\
& Negative & 10 & 22 & \\
& Mean score & $71 \%$ & $64 \%$ & \\
${ }^{*} \chi^{2}$ test. & & &
\end{tabular}

range 22 to 214 weeks). The mean progression-free survival time was 204 days (29 weeks, range 9 to 91 weeks). According to the log-rank test, we found a significant association between a Karnowski performance scale score (KPS) of at least $80 \%$ and a longer time interval to tumour progression (progression-free survival time; $\mathrm{p}=0.049$ ). There was no correlation between KPS $\geqslant 80$ and total survival time $(p=0.8)$ or between KPS $\geqslant 90$ and survival (progression-free survival time: $p=0.61$; total survival time: $p=0.7$ ). The following clinical variables also had no significant influence on patient survival (progressionfree survival time; total survival time): age $(\mathrm{p}=0.1 ; \mathrm{p}=0.2)$, $\operatorname{sex}(p=0.6 ; p=0.9)$, and chemotherapy $(p=0.5 ; p=0.8)$. The patient data are shown in table 1.

\section{Immunohistochemical expression of initial tumours and first recurrences}

As shown in table 2, we found different expression profiles for mdm2, EGFR, and msh2 in initial $v$ recurrent glioblastoma multiforme. Additionally, the Wilcoxon test identified significant differences in immunohistochemical scores for p53 $(p<0.0001)$ and msh2 $(p=0.0012)$, again with reduced values in tumour recurrences. We observed no association between immunohistochemical expression indices and survival (progression-free survival time, total survival time). In this series, we found no significant association between p53/mdm2/EGFR/msh2 expression and age (patients $<56$ years $v$ patients $\geqslant 56$ years of age; data not shown). Selected staining results for $\mathrm{p} 53, \mathrm{EGFR}$, and $\mathrm{msh} 2$ in initial $v$ recurrent glioblastoma multiforme are shown in figs $1-3$.

\section{Immunohistochemistry and chemotherapy}

We compared the $\mathrm{p} 53 / \mathrm{mdm} 2 / \mathrm{EGFR} / \mathrm{msh} 2$ expression profiles in patients who received chemotherapy with those who did not. The use of chemotherapy was associated with reduced msh2 expression. There was no statistically significant difference in p53, mdm2, or EGFR expression. There was also no
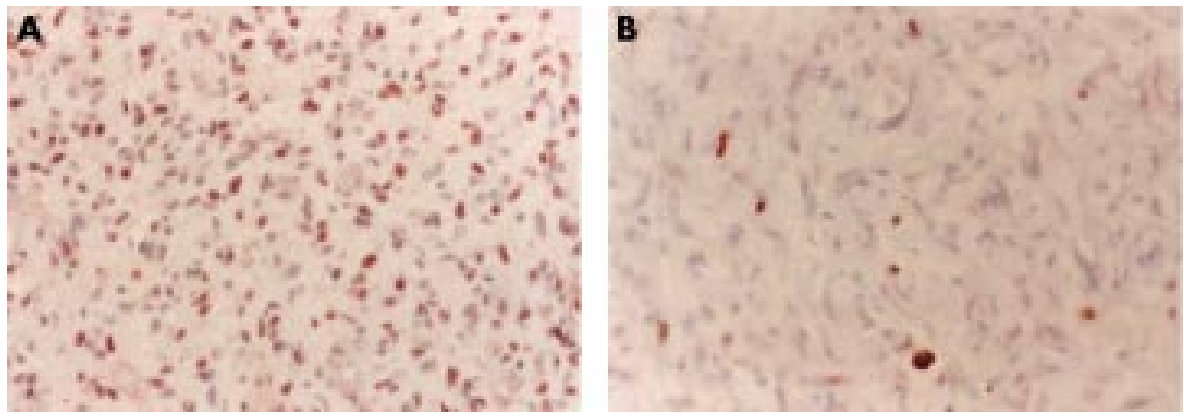

Figure 1 p53 downgrading in patient No 2: DO-1 nuclear protein expression in the initial presentation of glioblastoma multiforme (panel A: $36 \%$ positive tumour cells) and in recurrent tumour (panel B: $4 \%$ positive tumour cells). Magnification $\times 500$.
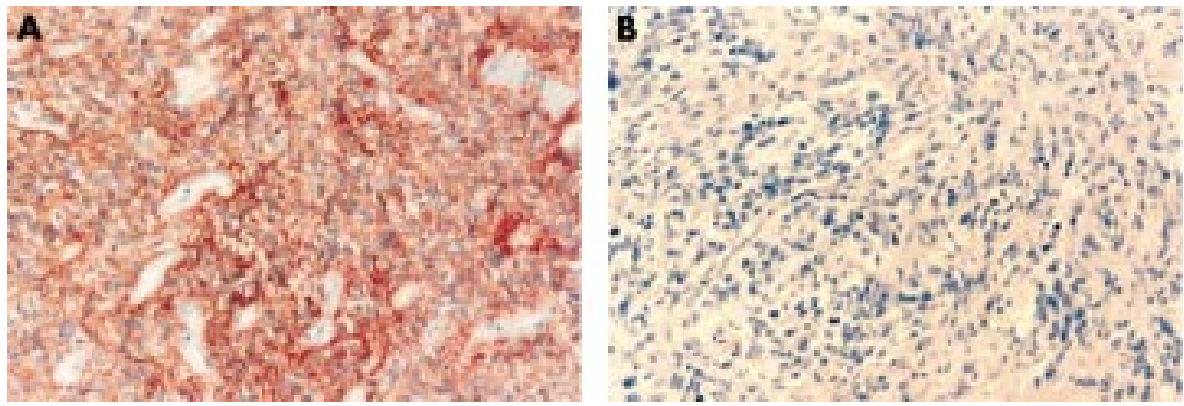

Figure 2 EGFR downgrading in patient $\mathrm{No}$ 2: $\mathrm{H} 11$ membrane staining in the initial tumour (panel $\mathrm{A}$ : positive tumour cells) and the recurrence (panel B: negative tumour cells. Magnification $\times 250$. 

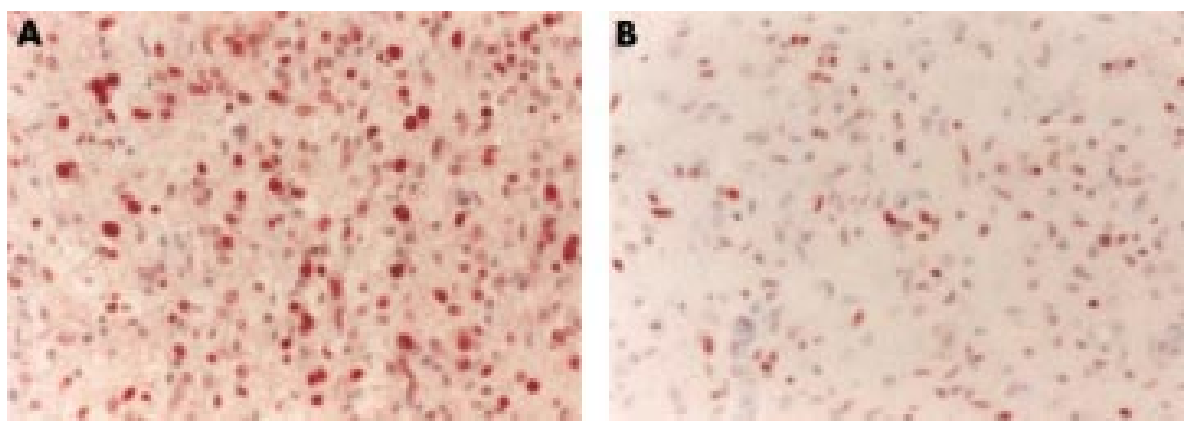

Figure 3 msh2 downgrading in patient No 12: $A B 1$ nuclear staining in the initial presentation of glioblastoma multiforme (panel $A: 67 \%$ positive tumour cells) $v$ the recurrent lesion (panel B: $40 \%$ positive tumour cells). Magnification $\times 500$.

\begin{tabular}{|c|c|c|c|c|c|c|c|}
\hline \multirow[b]{2}{*}{ Protein } & & \multicolumn{3}{|c|}{ No chemotherapy $(n=11)$} & \multicolumn{3}{|c|}{ Chemotherapy $(n=16)$} \\
\hline & & $\begin{array}{l}\text { Initial } \\
\text { tumoours }\end{array}$ & Recurrence & p Value* & $\begin{array}{l}\text { Initial } \\
\text { tumours }\end{array}$ & Recurrence & p Value* \\
\hline \multirow[t]{2}{*}{ p53 } & Positive & 10 & 9 & NS & 14 & 11 & NS \\
\hline & Negative & 1 & 2 & & 2 & 5 & \\
\hline \multirow[t]{2}{*}{$\mathrm{mdm} 2$} & Positive & 9 & 6 & NS & 14 & 11 & NS \\
\hline & Negative & 2 & 5 & & 2 & 5 & \\
\hline \multirow[t]{2}{*}{ EGFR } & Positive & 10 & $4 \dagger$ & $p=0.0002$ & 14 & $7 \dagger$ & $p=0.0004$ \\
\hline & Negative & 1 & 7 & & 2 & 9 & \\
\hline \multirow[t]{2}{*}{ msh2 } & Positive & 6 & 4 & NS & 11 & 1 & $p<0.0001$ \\
\hline & Negative & 5 & 7 & & 5 & 15 & \\
\hline
\end{tabular}

\begin{tabular}{|c|c|c|c|c|}
\hline Protein & & $\begin{array}{l}\text { Initial } \\
\text { GBM }\end{array}$ & 1st recurrence & $\begin{array}{l}\text { 2nd } \\
\text { recurrence }\end{array}$ \\
\hline p53 & $\begin{array}{l}\text { Positive } \\
\text { Negative }\end{array}$ & $\begin{array}{l}5 \\
2\end{array}$ & $\begin{array}{l}7 \\
0\end{array}$ & $\begin{array}{l}5 \\
2\end{array}$ \\
\hline \multirow[t]{2}{*}{$\mathrm{mdm} 2$} & Positive & 6 & 4 & 6 \\
\hline & Negative & 1 & 3 & 1 \\
\hline \multirow[t]{2}{*}{ EGFR } & Positive & 6 & 5 & 5 \\
\hline & Negative & 1 & 2 & 2 \\
\hline \multirow[t]{2}{*}{ msh2 } & Positive & 3 & 0 & 0 \\
\hline & Negative & 4 & 7 & 7 \\
\hline
\end{tabular}

GBM, glioblastoma multiforme.

significant difference in immunohistochemical scores as demonstrated by Wilcoxon testing. The data are given in table 3.

\section{Immunohistochemical expression in initial glioblastoma multiforme $v$ first and second recurrences}

In contrast to the above results, p53/mdm2/EGFR staining profiles of the seven paired specimens with first and second recurrences seemed more random. However, three of the seven initial glioblastoma multiforme specimens showed regular msh2 expression, whereas this protein was not detected in first or second recurrences. The data are given in table 4 .

\section{DISCUSSION}

From the results of this study, recurrent glioblastoma multiforme was characterised by the following immunohistochemical features:
- reduced amount of mdm2 and EGFR specimens $(\mathrm{p}=0.02$; $\mathrm{p}<0.0001)$

- reduced expression of msh2 ( $\mathrm{p}<0.0001)$;

- reduced immunohistochemical scores for p53 ( $p<0.0001)$ and msh2 $(\mathrm{p}=0.0012)$.

Three reasons for reduced immunostaining in recurrent lesions need to be considered: first, differentially expressed proteins might not be detected by the antibody used in the study; second, the expression level might actually decrease; third, recurrent glioblastoma multiforme might represent a tumour stage of generally reduced protein expression caused by further dedifferentiation.

In our study, the immunohistochemical expression of p53, mdm2, EGFR, and msh2 in initial glioblastoma multiforme was compared to that of recurrent lesions in the same patients. The study was limited in some ways: the patients were selected for re-craniotomy mainly by their performance status (this may also explain the fact that patient survival was not correlated with patient age); second, owing to the low number of patients undergoing craniotomy for glioblastoma multiforme recurrence in general, the number of specimens is small; finally, only small parts of the tumours were stained. Subclones of this tumour may have different biological behaviour and different expression patterns.

Many genetic alterations have been associated with the genesis of glioblastoma multiforme. ${ }^{256}$ In contrast, little has been published about immunohistochemical investigations in recurrent glioblastoma. Strik et al described upregulation of antiapoptotic proteins and a downregulation of the proapoptotic bcl-2 family member bax in recurrent $v$ initial glioblastoma multiforme. ${ }^{19}$ These differences were highly significant in patients treated with chemotherapy and radiotherapy but showed only a trend in untreated patients. Some investigators have reported an inverse correlation of Ki67 PI with progression-free survival time and total survival time in 
gliomas of all WHO grades. ${ }^{201}$ Deininger et al described a correlation between a high p53 score (detected by the mAb DO-1 which detects wild type as well as mutated p53) and a short time to tumour progression only in those patients who underwent radiochemotherapy. ${ }^{22}$ They furthermore found that radiation treatment led to a reduction in p53 scores (detected by the mAb DO- 7 which detects mainly mutated $\mathrm{p} 53$ protein) and an increase in bcl-2 scores.

A decrease in DO- 1 detected p53 expression, as demonstrated by Wilcoxon testing, could indicate a decrease in wildtype p53 in recurrent tumours. In comparison with the study by Deininger et al, all of our patients received radiotherapy and most of them underwent additional chemotherapy. However, we found no significant association between DO-1 detected p53 expression and progression-free survival time. With regard to the fact that p53 induces mdm2 transcription, mdm2 protein levels could be reduced because of decreased p53 protein expression. On the other hand, many splicing variants have been described for $\mathrm{mdm} 2$ protein. ${ }^{23}$ These could be differentially expressed in different stages of the disease. However, there was no significant association between p53/mdm2 expression and survival. EGFR has been described as an important factor in glioblastoma growth. ${ }^{2}$ We observed reduced expression in recurrent tumours but no significant association between EGFR detection or EGFR loss and survival.

In agreement with the results of Friedman et al, ${ }^{11} \mathrm{msh} 2$ was expressed in the majority of glioblastoma multiforme specimens. Those investigators furthermore reported no significant association between the msh2 expression and the response of glioblastoma and anaplastic astrocytoma to temozolomide chemotherapy. We found that the administration of chemotherapy (mainly cisplatin/tamoxifen) was associated with reduced expression of $\mathrm{msh} 2$ in recurrent lesions $(\mathrm{p}<0.0001)$. It should be noted that temozolomide chemotherapy $(\mathrm{n}=1)$ was given after re-craniotomy for tumour recurrence and thus could not have had any influence on the immunohistochemical expression profile.

\section{Conclusions}

Bearing in mind the limitations listed above, recurrent glioblastoma multiforme is characterised by significantly reduced p53/mdm2/EGFR/msh2 expression. The administration of chemotherapy was associated with reduced expression of the MMR protein msh2. Our results indicate that significant changes occur during the life span of patients suffering from glioblastoma. However, neither p53/mdm2/EGFR/msh2 expression nor reduced expression in recurrent glioblastoma multiforme was associated with survival.

\section{Authors' affiliations}

A M Stark, P Witzel, R J Strege, H-H Hugo, H M Mehdorn, Department of Neurosurgery, University of Kiel Medical Centre, Kiel, Germany

Competing interests: none declared

\section{REFERENCES}

1 Hurtt MR, Moossy J, Donovan-Peluso M, et al. Amplification of epidermal growth factor receptor gene in gliomas: histopathology and prognosis. J Neuropathol Exp Neurol 1992;51:84-90.

2 Kleihues P, Cavenee WK. Pathology and genetics of tumours of the nervous system. Lyon: IARC Press, 2000.

3 Schiebe $M$, Ohneseit $P$, Hoffmann W, et al. Analysis of mdm2 and p53 gene alterations in glioblastomas and its correlation with clinical factors. J Neurooncol 2000;49:197-203.

4 Sehgal A. Molecular changes during the genesis of human gliomas. Semin Surg Oncol 1998;14:3-12.

5 von Deimling A, Louis DN, Wiestler OD. Molecular pathways in the formation of gliomas. Glia 1995;15:328-38.

6 Kleihues P, Ohgaki H. Primary and secondary glioblastomas: from concept to clinical diagnosis. Neuro-oncology 1999;1:44-51.

7 Scherer HJ. Cerebral astrocytomas and their derivatives. Am J Cancer 1940;40: 159-98.

8 von Deimling A, Fimmers R, Schmidt MC, et al. Comprehensive allelotype and genetic analysis of 466 human nervous system tumours. Neuropathol Exp Neurol 2000;59:544-58.

9 Balint E, Vousden $\mathrm{KH}$. Activation and activities of the p53 tumour suppressor protein. Br J Cancer 2001;85:1813-23.

10 Michael D, Oren M. The p53 and mdm2 families in cancer. Curr Opin Genet Dev 2002;12:53-9.

11 Friedman HS, McLendon RE, Kerby T, et al. DNA mismatch repair and O6- alkylguanine-DNA alkyltransferase analysis and response to temodal in newly diagnosed malignant glioma. J Clin Oncol 1998;16:3851-7.

12 Kolodner RD, Marsischky GT. Eukaryotic DNA mismatch repair. Curr Opin Genet Dev 1999;9:89-96.

13 Leung SY, Chan TL, Chung LP, et al. Microsatellite instability and mutation of DNA mismatch repair genes in gliomas. Am J Pathol 1998;153:1181-8.

14 Nieder C, Petersen S, Petersen C, et al. The challenge of P53 as prognostic and predictive factor in gliomas. Cancer Treat Rev 2000;26:67-73

15 Simmons ML, Lamborn KR, Takahashi M, et al. Analysis of complex relationships between age, p53, epidermal growth factor receptor, and survival in glioblastoma patients. Cancer Res 2001;61:1122-8.

16 Newcomb EW, Cohen H, Lee SR, et al. Survival of patients with glioblastoma multiforme is not influenced by altered expression of $\mathrm{pl6}$, p53, EGFR, MDM2 or Bcl-2 genes. Brain Pathol 1998;8:655-67.

17 Velasco A, Hewitt SM, Albert PS, et al. Differential expression of the mismatch repair gene hMSH2 in malignant prostate tissue is associated with cancer recurrence. Cancer 2002;1:690-9.

18 Reifenberger J, Ring GU, Gies U, et al. Analysis of p53 mutation and epidermal growth factor receptor amplification in recurrent gliomas with malignant progression. J Neuropathol Exp Neurol 1996;55:822-31.

19 Strik H, Deininger M, Streffer J, et al. Bcl-2 family protein expression in initial and recurrent glioblastomas: modulation by radiochemotherapy. $J$ Neurol Neurosurg Psychiatry 1999;67:763-8.

20 Enestrom S, Vavruch L, Franlund B, et al. Ki-67 expression as a prognostic factor in primary and recurrent astrocytomas. Neurochirurgie 1998;44:25-30.

21 Ralte AM, Sharma MC, Karak AK, et al. Clinicopathological features, MIB-1 labeling index and apoptotic index in recurrent astrocytic tumors. Pathol Oncol Res 2001;7:267-78.

22 Deininger $\mathbf{M H}$, Grote E, Wickboldt J, et al. Distinct radiochemotherapy protocols differentially influence cellular proliferation and expression of p53 and bcl-2 in glioblastoma multiforme relapses in vivo. J Neurooncol 2000;48:121-9.

23 Olson DC, Marechal V, Momand J, et al. Identification and characterization of multiple $\mathrm{mdm}-2$ proteins and $\mathrm{mdm}-2-\mathrm{p} 53$ protein complexes. Oncogene 1993;8:2343-60. 